
\title{
R Reserach S Suare \\ KLF4 Inhibits Activation of Human Hepatic Stellate Cells LX-2 in vitro
}

\author{
YinKai Xue \\ Wuhan Union Hospital \\ Jun TAN \\ Wuhan Union Hospital \\ TianCi Yao \\ Wuhan Union Hospital \\ QingYong Chen \\ Wuhan Union Hospital \\ Ke Jiang \\ Wuhan Union Hospital \\ ZhiRui Wang \\ Wuhan Union Hospital
}

Hai Zheng ( $\sim$ zhenghai_xiehe@126.com )

Wuhan Union Hospital https://orcid.org/0000-0002-2427-8897

Research

Keywords: Kruppel-like factor 4 (KLF4 ), HSC, hepatic stellate cell activity, LX-2 cells, liver fibrosis

Posted Date: September 7th, 2021

DOI: https://doi.org/10.21203/rs.3.rs-702048/v1

License: (c) (i) This work is licensed under a Creative Commons Attribution 4.0 International License.

Read Full License 


\section{Abstract}

\section{Background}

Hepatic Stellate Cell (HSC) represents a key factor in liver fibrosis. Early-stage liver fibrosis is still reversible and is intimately associated with the state of HSC. KLF4 has been shown to play a pivotal role in a wide array of physiological and pathological processes.

\section{Methods}

In this study, we examined the effect of Kruppel-like factor 4 (KLF4) on the proliferation, apoptosis and phenotype of HSC in resting state in human HSC LX-2 cells, and the role KLF4 plays in the maintenance of the resting state of HSC, with an attempt to provide an experimental basis for the diagnosis, treatment and prognosis evaluation of liver fibrosis. We designed a KLF4 lentiviral vector and a KLF4 shRNA lentiviral vector, to up-regulate and silence KLF4 expression in LX-2 cells by transfection. We examined LX-2 cell proliferation by plate cloning and CCK8 assay, flow cytometrically detected cell cycle distribution and cellular apoptosis rate and determined some quiescence and activation markers of HSC by Western blotting.

Results

Our result showed that E-cadherin and ZO-1, dubbed quiescent HSC markers, were significantly increased. N-cadherin while a-AMA, known as activated HSC marker, was significantly decreased. In contrast, cell proliferation and apoptosis rate were elevated in LX-2 cells whose KLF4 expression had been silenced.

\section{Conclusions}

Our results indicated that KLF4 inhibited the proliferation and activation of human HSC LX-2 and might be a key regulatory protein in the maintenance of quiescence of HSC and might serve as a target for the inhibition of hepatic fibrosis.

\section{Introduction}

Hepatic stellate cells (HSC), or fat-storing cells, are normally localized in the perisinusoidal space and produce only a small amount of extracellular matrix (ECM) components for the formation of the basement membrane(Suarez-Cuenca et al., 2008). When exposed to soluble factors or changes in matrix stiffness (Suarez-Cuenca et al., 2008), they morphologically transform to myofibroblast-like cells (Anthony et al., 2010; Sako et al., 1987; Shields et al., 1996). In the trans-differentiation they lose retinoid stores and acquire a myofibroblast phenotype, thereby becoming highly proliferative and migratory and having type I collagen deposition in the parenchyma(Garrett-Sinha et al., 1996). Transforming growth factor- $\beta$ (TGF- $\beta$ ) is a central regulator in chronic liver disease and is implicated in all stages of disease, ranging from initial liver injury to inflammation and, eventually, fibrosis. Mounting 
evidence showed that HSC plays a pivotal part in the development of hepatic fibrosis, i.e., Quiescent HSCs, after being activated by TGF- $\beta /$ Smad3/ Snail1 signalling pathway, turned into myofibroblasts and then produce fibrous scars, a characteristic feature of liver cirrhosis.

Kruppel-like factor 4 (KLF4) is a recently cloned transcriptional factor and is highly expressed in the gastrointestinal tract and other epithelial tissues, and is involved in the regulation of cell differentiation, maturation and inhibition. Recent findings suggest that KLF4 contributed to the regulation of proliferation, migration, differentiation and inhibition of epithelial-to-mesenchymal transition (EMT) in iPS (induced pluripotent stem cells) cells, breast cancer cells and lung fibroblast cells by inhibiting TGF$\beta /$ Smad3/Snail signaling pathway. Whether KLF4 is involved in the inhibition of HSC activation in liver fibrosis remains poorly understood.

However, reports on the relationship between KLF4 and hepatic stellate cells and liver fibrosis are scanty. Our previous study found that the TGF- $\beta$-induced expression of KLF4 mRNA was significantly downregulated in LX-2 cells. Transforming growth factor- $\beta$ (TGF- $\beta$ ) is generally believed to promote liver fibrosis. Multiple studies demonstrated that TGF- $\beta$ is the most important pro-fibrogenic damage stimulating factor in the liver (Forbes \& Parola, 2011; Guyot et al., 2006; Moreira, 2007). TGF- $\beta$ inhibits HSC apoptosis, increases the number of activated HSC (George et al., 1999), and induced over-production of extracellular matrix by sinusoidal endothelial cells (George et al., 2000). Animal studies exhibited that therapeutic strategies involving TGF- $\beta$ signaling pathway significantly reduced liver fibrosis. HSC activation acts as the central link in the process of liver fibrosis (Anthony et al., 2010; Zhao et al., 2013). TGF- $\beta$ can significantly reduce the expression of KLF4 in LX-2 cells, suggesting that KLF4 may serve as an important modulator of HSC activation and thereby regulate the liver fibrosis. Therefor, we were led to speculate that KLF4 might be a regulator that controls the activation of resting $\mathrm{HSC}$, and might be used as a molecular target for the inhibition of liver fibrosis progression.

\section{Materials And Methods}

\subsection{Primary reagents and Cells}

DMEM/High-sugar medium was bought from HyClone, and fetal bovine serum (FBS) was from Gibco. Penicillin/streptomycin double antibody was procured from Hydone. Diethypyrocarbonate (DEPC), TritonX, DAPI (4, 6-diamidino-2-phenylindole) and crystal violet were products of Sigma. PCR primer and Trizol Reagent came from Invitrogen. Transwell Chamber was produced by BD. E-cadherin antibody, vimentin antibody, Z0-1 antibody, KLF4 antibody and Snail antibody were all from CST. Lentivirus-KLF4+, Lentivirus-Con+, Lentivirus-siKLF4 and Lentivirus-siCon were purchased from ShangHai JiKai. Flow cytometry buffer was from eBioscience. RNA transcription kit and SYBR Green Real-time PGR Mix were bought from Takara. GAPDH antibody was procured from Wuhan Boshide. CCK-8 kit was from DOJINDO. PMSF, RIPA Lysate and BCA Protein concentration assay kit came from Biyuntian. Human hepatic stellate cells LX-2 was kindly provided by Dr. Leng Zhengwei.

\subsection{Reagent preparation}


Preparation of phosphate buffer saline: $\mathrm{NaCl} 8.00 \mathrm{~g}, \mathrm{KCl} 0.4 \mathrm{~g}, \mathrm{Na} 2 \mathrm{HPO} 40.78 \mathrm{~g}$, Na2HPO4 $3.6 \mathrm{~g}$ were put in $700 \mathrm{ml}$ of triple-distilled water, fully stirred, allowed to dissolve, with $\mathrm{pH}$ set at 7.4. The result sample was added to a final volume of $1000 \mathrm{ml}$ with triple-distilled water and then routinely autoclaved.

Preparation of $0.25 \%$ trypsin (total volume $200 \mathrm{ml}$ ): $0.5 \mathrm{~g}$ of trypsin, $0.4 \mathrm{~g}$ of glucose and $0.04 \mathrm{~g}$ of EDTA were put into $200 \mathrm{ml}$ of PBS, stirred gently and slowly, allowed to dissolve, and filtered through a $0.22 \mu \mathrm{m}$ filter on a clean bench.

Preparation of complete medium (total volume $100 \mathrm{ml}$ ): Under sterile conditions, $90 \mathrm{ml}$ of DMEM highsugar medium stock solution, $10 \mathrm{ml}$ of Gibco fetal bovine serum, $1 \mathrm{ml}$ of penicillin/streptomycin were put into a sterile bottle and fully mixed. The samples were labeled and stored at $4^{\circ} \mathrm{C}$.

0.1\% crystal violet alcohol solution: $50 \mathrm{mg}$ crystal violet was dissolved in $10 \mathrm{ml}$ absolute ethanol to prepare $0.5 \%$ mother liquor, and the mother liquor and physiological saline were mixed at 1:4 to obtain $0.1 \%$ crystal violet staining solution.

0.1\% DEPC water: $100 \mu \mathrm{l}$ DEPC was added to $100 \mathrm{ml} \mathrm{ddH} 2 \mathrm{O}$, allowed to dissolve at $37^{\circ} \mathrm{C}$, and shaken at $80 \mathrm{rmp}$ overnight.

75\% ethanol: Absolute ethanol was mixed with DEPC water at 3:1 and stored at $-20^{\circ} \mathrm{C}$ for later use.

\subsection{Cell Culture}

LX-2 cells were cultured in a complete medium under saturated humidity, at $37^{\circ} \mathrm{C}$ and in $5 \% \mathrm{CO} 2$. Under a microscope, when the cell density reached $70-80 \%$, the culture solution was discarded. Then trypsin $(0.25 \%$ trypsin $+0.02 \%$ EDTA) or PBS was added and the sample was gently rinsed once, and the rinsing solution was thrown away. Afterwards, trypsin-digested cells were put into the cell culture incubator (digestion time varied, usually lasting 1 5 min). Then, complete medium was added to terminate digestion, evenly blow. Then the sample was centrifuged at $1000 \mathrm{rpm}$ for $5 \mathrm{~min}$ to collect appropriate amount of cells. continue to culture. Cells at the logarithmic growth phase with good growth state were taken, and then suspended in a frozen solution (90\% FBS + 10\% DMSO). After routine digestion, the sample was stored at $4{ }^{\circ} \mathrm{C}$ for $30 \mathrm{~min}$ at $-20^{\circ} \mathrm{C}$ for $60 \mathrm{~min}$, and then preserved at $-80^{\circ} \mathrm{C}$. The samples were stored in a liquid nitrogen tank for over 2 months. Then, the frozen cells were taken out and quickly placed in a $37^{\circ} \mathrm{C}$ water bath. After being thawed, they were centrifuged at $800 \mathrm{rpm}$ for $5 \mathrm{~min}$. The supernatant was discarded and the cells were suspended in a 10\% FBS medium and incubated. The counting plate was rinsed with double-distilled water, with the residues removed, and and absorbed with absorbent paper. A new coverslip was taken in the middle of the counting plate. Single cells were isolated and Trypan blue staining solution was mixed suspension at 1:1 (v/v). After full mixing, $20 \mu \mathrm{l}$ of the mixed suspension was taken and carefully put between the counting plate and the coverslip, and the samples were left undisturbed until the cell suspension was evenly distributed. Under a 10x inverted microscope, the living cells were unstained, and the non-viable cells were stained blue.

\subsection{RNA extraction, reverse transcription and real-time PCR}


RT-PCR can quantitatively detect the mRNA expression level of a certain gene in real time.

Methodologically, the RNA extracted from the sample is added to a specific primer and then reversely transcribed into cDNA for real-time PCR reaction. With RT-PCR, the reaction product is detected by SYBR Greenl. The fluorescent SYBR Greenl can bind to double-stranded DNA. SYBR Greenl combined with double-stranded DNA is separated from the double-stranded DNA, and the fluorescent signal is released and detected on a PCR machine, and the amount of DNA synthesis is calculated according to fluorescein intensity detected. PCR was performed on the following thermal cycling profile: 45 cycles of denaturation at $95^{\circ} \mathrm{C}$ for $30 \mathrm{~s}$, annealing at $60^{\circ} \mathrm{C}$ for $60 \mathrm{~s}$, and extension at $95^{\circ} \mathrm{C}$ for $5 \mathrm{~s}$. For each sample, reactions were duplicated and the average mRNA level of each gene was determined by using the $\Delta \Delta \mathrm{Ct}$ method. The primer pairs for measuring the levels of each gene are listed as below.

KLF4-Forward CGAACCCACACAGGTGAGAA

KLF4-Reverse TACGGTAGTGCCTGGTCAGTTC

GAPDH-Forward GGGGAGCCAAAAGGGTCATCATCT

\section{GAPDH-Reverse GACGCCTGCTTCACCACCTTCTTG}

\subsection{Western Blot Analysis}

Protein extracts were electrophoresically separated on SDS-polyacrylamide gels of various concentrations, depending on the expected sizes of measured proteins, and were then transferred to PVDF membranes (Millipore, USA). After blockade with 5\% nonfat milk and $0.1 \%$ Tween 20 in TBS for $1 \mathrm{~h}$, the membrane was then incubated, at $4{ }^{\circ} \mathrm{C}$ overnight, with the primary antibodies and then with goat antirabbit secondary antibodies. Primary antibodies used in this analysis included: rabbit anti-E-cadherin, rabbit anti-N-cadherin, rabbit anti-ZO-1, and rabbit anti- a-SMA, rabbit anti-KLF4 antibodies (Santa Cruz Biotec).

\subsection{Colony Formation Assay}

Colony formation assay was performed for adherent LX-2 cells as previously described (Novo et al., 2014). Briefly, single cells (500 cells per well) were plated into 6-well plates and cultured for 10 days. After stained with violet, cells were photographed and examined for their proliferation and colony formation efficiency. All the experiments were performed at least three times.

\subsection{Lentiviral Infection of LX-2}

LX-2 cells at logarithmic growth phase were routinely digested, put into complete medium to make singlecell suspension and counted. $1 \times 107$ cells per well were suspended in $3 \mathrm{ml}$ complete medium, and cultured in an incubator for 12 16 h, until the cells reattached to the wall (LX-2 proliferation time is $17 \sim 24 \mathrm{~h}$ ). At $1 \mathrm{ml}$ complete medium per well, with $\mathrm{MOI}$ (virus number/cell number) $=10$, the cells were transfected with corresponding amount of lentivirus and were cultured for $24 \mathrm{~h}$. 


\subsection{Annexin-V APC/PI method for apoptosis detection}

Cell were collected, washed with phosphate-buffered saline (PBS) and stained with Annexin-V APC (Keygen, China) and PI (Sigma) both for $10 \mathrm{~min}$ at room temperature in the dark according to the manufacturer's instructions. All stained cells were then flow cytometrically examined.

\subsection{CCK-8 assay}

CCK-8 assay was used to determine the effect of KLF4 on the proliferation of human LX-2 cells. (CCK-8 assay kit , DOJINDO, molecular technolonies, Inc, Japan) by following manufacturer's instructions. Briefly, 5,000 cells were seeded into each well of 96 -well plates. 7 wells were inoculated with each cell, and another 2 wells were added with the cell-free complete medium. All the wells were incubated at $37^{\circ} \mathrm{C}$ and in $5 \% \mathrm{CO} 2$ in a humidified condition for 12-16 hours. The absorption of each well was read at $450 \mathrm{~nm}$ on a plate reader. Cell viability was calculated as follows: Absorption of experiment-Absorption of blank)/(Absorption of control-Absorption of blank) $\times 100 \%$.

\subsection{Statistical Analysis}

Data obtained were expressed as mean \pm standard deviation, and Student's $t$ test was used. A P $<0.05$ was considered to be statistically significant.

\section{Results}

\subsection{KLF4 mRNA expression in LX-2 cells cultured with different concentrations of TGF- $\beta$ was decreased after 24 hours.}

After the human hepatic stellate cell line LX-2 were cultured with different concentrations of TGF- $\beta$ for 24 hours, RT-PCR showed that KLF4 mRNA level was significantly decreased $(P<0.05)($ fig. 1$)$.

\subsection{The expression of KLF4 in LX-2 cells transfected with KLF4 lentiviral vector and KLF4 siRNA lentiviral vector experienced significant change.}

KLF4 lentiviral vector and siRNA lentiviral vector labeled with enhanced green fluorescence (eGFP) were used to transfect human hepatic stellate cell line LX-2 in vitro, and KLF4 expression was up-regulated and down-regulated, respectively (fig.2) after the transfections.

LX-2 cells were transfected with empty virus and cultured for 10 days (fluorescence imaging). D: KLF4 overexpression group: LX-2 cells were transfected with KLF4 and cultured for 10 days (fluorescent imaging). E: KLF4 down-regulation group. LX-2 cells were transfected with empty virus and cultured for 10 days (fluorescence imaging). F: KLF4 down-regulation group. LX-2 cells were transfected with siRNA virus and cultured for 10 days (fluorescence imaging).

\subsection{The proliferation of LX-2 cells after KLF4 treatment was significantly changed.}


KLF4 lentiviral vector was transfected into LX-2 cells. CCK8 detection showed showed that the upregulated KLF4 expression significantly inhibited the proliferation of $L X-2$ cells $(P<0.05)$. After transfection with siRNA lentiviral vector, CCK8 detection revealed that the proliferation of LX-2 cells was significantly enhanced after KLF4 expression was silenced $(P<0.05)(f i g .3)$.

A . Effect of KLF4 overexpression on LX-2 cell proliferation B. Effect of inhibited KLF4 expression on LX-2 cell proliferation

\subsection{Significant changes in cell proliferation ability after KLF4 intervention.}

KLF4 lentiviral vector was transfected into LX-2 cells. Plate cloning exhibited that the number of clones per unit area of LX-2 cells was significantly decreased after KLF4 expression was up-regulated $(P<0.05)$. siRNA lentiviral vector was transfected into LX-2 cells. The plate cloning showed that the number of clones per unit area of LX-2 cells was increased significantly after KLF4 expression was silencing $(P<0.05)$ (fig. 4).

A. KLF4 overexpression group, empty virus was transfected into LX-2 cells. B. KLF4 overexpression group, KLF4 virus was transfected into LX-2 cells. C. KLF4 down-regulation group, empty virus was transfected into LX-2 cells.D. KLF4 down-regulation group, siRNA virus was transfected into LX-2 cells. E. KLF4 overexpression group: plate cloning. F. KLF4 down-regulation group: plate cloning.

\subsection{After up-regulation of KLF4 expression, the number of $L X-2$ cells in $G_{0} / G_{1}$ phase and $S$ phase was increased.}

KLF4 lentiviral vector was transfected into LX-2 cells, which up-regulated KLF4 expression. Flow cytometry showed that overexpression of KLF4 in LX-2 cells significantly increased G0-G1 phase cells $(P<0.05)$. The number of $S$ phase cells was significantly reduced, and no significant change was found in the number of $\mathrm{G} 2 / \mathrm{M}$ phase cells (fig. 5, table 1 ).

Table 1 Effect of KLF4 on cell cycle of human hepatic stellate cell LX-2

Cell cycle (\%)

Group
$\mathrm{G}_{0} / \mathrm{G}_{1}$
S
$\mathrm{G}_{2} / \mathrm{M}$

LX-2 control group

$48.87 \pm 1.22 \quad 46.21 \pm 1.34$

$4.62 \pm 2.01$

KLF4 over-expression in LX-2

$61.88 \pm 2.10 * 36.04 \pm 1.78 *$

$5.08 \pm 1.67$

\subsection{After KLF4 intervention, LX-2 cells experienced significant changes in apoptosis.}


KLF4 lentiviral vector was transfected into LX-2 cells, which up-regulated KLF4 expression. Flow cytometry showed that the apoptosis of LX-2 cells overexpressing KLF4 was decreased at both the early and late stages $(P<0.05)$. siRNA lentiviral vector was transfected into $L X-2$ cells, which down-regulated KLF4 expression. Flow cytometry exhibited that the apoptosis of LX-2 cells with KLF4 down-regulated by silencing was increased at the early stages $(P<0.05)$ (fig 6. fig 7).

A. KLF4 up-regulation group: Flow cytometrical results of LX2 cells transfected with empty virus. B. KLF4 up-regulation group: Flow cytometrical results of LX-2 cells overexpressing KLF4. UL stands for early apoptotic cells and UR for late apoptotic cells.

A. KLF4 inhibition group: LX-2 cells transfected with empty virus (control). B. KLF4 inhibition group: LX-2 cells with KLF4 down-regulation. UL stands for early apoptotic cells and UR for late apoptotic cells.

\subsection{LX-2 cells overexpressing KLF4 underwent significant changes in indicators of quiescence and activation states.}

KLF4 lentiviral vector was transfected into LX-2 cells, which up-regulate KLF4 expression. Western blotting that the expression of HSC quiescence-associated proteins, i.e., E-cadherin and ZO-1, was upregulated while the expression of HSC activation-associated proteins, i.e., a-SMA and N-cadherin was down-regulated $(P<0.05)$ (fig. 8).

A. Western blot bands B. Western blotting chart

\section{Discussion}

Hepatic fibrosis is a chronic pathological change of the liver caused by the simultaneous injury and repair of the liver in the presence of chronic injury factors. The process is characterized by the abnormal deposition of matrix components in the liver, which further triggers aberrant structural and functional changes and thereby leads to progression of chronic liver disease to cirrhosis. The mechanism of liver fibrosis is multi-factorial, and is associated with alcoholic liver disease, viral hepatitis, autoimmune diseases, fatty liver, among others. Multiple studies have confirmed that the core event of the liver fibrosis is the activation of hepatic stellate cells (HSC) (Zhao et al., 2013). Activated HSC promotes selfproliferation and synthesizes a large amount of extracellular matrices that deposits in the liver, through autocrine and paracrine effects, eventually resulting in liver fibrosis.

Hepatic stellate cells (HCS), also known as Ito cells, lipocytes or perisinusoidal cells, are predominantly located in the Divine's space, between the liver cells and the sinusoidal endothelium, and are rich in vitamin A lipid droplets. They are the main source of myofibroblasts during injury (about $70-80 \%$ ). Other sources of HSC include: hilar fibroblasts (Jhandier et al., 2005; Wells et al., 2004), hematopoietic stem cell-derived fibroblasts (Kisseleva et al., 2006), bone marrow (Forbes et al., 2004) and epithelial stromal cell transformation (Zeisberg et al., 2007). Under physiological conditions, hepatic stellate cells are at quiescent state. HSCs in quiescent (resting) state showed low proliferative capacity and low fibrosis 
tendency, and virtually secreted no cytokines and lacked contractility (Maher et al., 1994; Rockey et al., 1993). Their principal function to store and metabolize vitamin A lipid droplets (Blomhoff \& Wake, 1991). When liver fibrosis progresses, the HSC phenotype is constantly altering with the change in the microenvironment, such as decreased cell junctions, increased migration capacity, elevated a-SMA expression in HSC, reduced vitamin A lipid droplets, and transdifferentiation into myofibroblasts. which are activated (Senoo et al., 2010; Tsukamoto, 2005).

The activation of hepatic stellate cells mainly consists of two phases: the initiation phase and the duration (Minar, 1987). The initiation phase, also known as the pro-inflammatory phase, is characterized by early gene expression and phenotypic changes in HSC, resting state-specific markers, i.e., glial fibrillary acidic protein (GFAP) and peroxisome proliferator-activated receptor (PPAR- $y$ ), ZO-1, E-cadherin, vitamin A lipid droplets are loss, original stellate cells morphological changes, showing migration and differentiation and proliferation. "Duration" is also known as continuous activation phase, including at least seven HSC biological behavioral changes in this stage: cell proliferation, chemotaxis, fibrosis, contractility, changes in matrix degradation, loss of retinoids, and changes in inflammatory signals (Ghiassi-Nejad \& Friedman, 2008). When stimulation persists, HSC proliferates, transforms into myofibrolasts, expresses activation markers, such as N-cadherin, a-SMA, desmin and secretes a large amount of ECM (Eng \& Friedman, 2000; Friedman, 2000), which is involved in the liver fibrosis. When stimulatory factors are removed, the activated HSC can be cleared by the apoptotic mechanism or reconverted to resting HSC (Lee \& Friedman, 2011).

This study found that HSC activation and differentiation into myofibroblasts is the core event of the liver fibrosis (Anthony et al., 2010; Novo et al., 2014; Zhao et al., 2013). On the one hand, activated HSC participates in the remodeling of intrahepatic structures and the formation of HF by self-proliferation and secretion of extracellular matrix components. On the other hand, the hepatic sinus pressure is increased due to cell contraction, and these two changes morphologically and pathologically underlie the pathogenesis of liver fibrosis and cirrhosis.

Gressner et al (Gressner et al., 2002) found that TGF- $\beta$ is the most important regulatory factor that induces the transformation of HSC into myofibroblasts, increases the level of activation markers, and upregulates collagen expression. Domitrović $\mathrm{R}$ et al. showed that excessive ECM could cause hepatocyte damage (Domitrovic et al., 2009), and the hepatic parenchymal damage triggers HSC activation. At the same time, activated HSCs differentiated into myofibroblasts, which can abundantly secrete collagen and migrate to necrotic and inflammatory areas. Excessive deposition of ECM can damage liver cells and affect their metabolic function (Domitrovic et al., 2009). Therefore, HSC plays an important role in the development of liver fibrosis. HSC is a major source of tissue metalloproteinase inhibitors, which can inhibit matrix metalloproteinase activity and regulate the balance between synthesis and degradation of ECM (Ramachandran \& Iredale, 2012). Fibrosis is characterized by excessive accumulation of the extracellular matrix (ECM) when the imbalance was upset between synthesis and degradation of ECM (Schnaper, 1995). HSCs release a variety of cytokines that regulate gene expression and cell phenotypes at the early stages of fibrosis via paracrine pathway (Heymann et al., 2009). Both experimental and 
clinical studies demonstrated that reduced number of activated HSC is an important indicator of liver fibrosis reversal, and the self-repair from liver fibrosis mainly relies on apoptosis of activated HSC(Gonzalez et al., 2009; Suarez-Cuenca et al., 2008). The early fibrosis is reversible, and myofibroblasts can be re-transformed to an inactive state during the reversal of liver fibrosis (Kisseleva et al., 2012). Activated HSCs play an important role in the mediation of liver immune response. Stellate cells produce a series of chemotactic peptides (especially chemokines), which amplify the inflammatory effects of inflammatory cells and also directly interact with lymphocyte subsets, including natural killer cells, to participate in the inflammatory process (Wang et al., 2013). These studies suggested that HSC is not only the central link in the progression of liver fibrosis, but also may serve as target for reversing liver fibrosis.

Krüppel-like factor 4 (KLF4) is a eukaryotic zinc finger protein transcription factor that is widely expressed in various tissues of mammals and can bidirectionally regulate the activation and inhibition. It plays an important role in cellular proliferation, apoptosis, migration, differentiation, phenotype and embryo development.

\section{Conclusion}

The expression level of KLF4 mRNA in LX-2 cells cultured with transforming growth factor- $\beta$ (TGF- $\beta$ ) was decreased significantly. Transforming growth factor- $\beta$ (TGF- $\beta$ ) induced a significant decrease in KLF4 mRNA expression in cultured LX-2 cells. TGF- $\beta$ is widely believed to promote liver fibrosis, and hepatic stellate cell activation is a central event of the liver fibrosis, suggesting that there might be a relationship between KLF4 and HSC activation. After overexpression of KLF4, LX-2 cell proliferation was inhibited and apoptosis was reduced. After silencing KLF4 expression, LX-2 cells proliferation was enhanced and early apoptosis increased.

After KLF4 expression was up-regulated, the expression of epithelial markers E-cadherin and ZO-1 in LX-2 cells were increased, and the expression of interstitial markers $\mathbb{B}$-SMA, N-cadherin and vimentin were decreased. These findings confirmed that KLF4 can inhibit the proliferative ability of activated LX-2 cells and promote the change of activated LX-2 cells back to resting state. These results led us to theorize that KLF4 may be a functional regulator of quiescent HSC and may serve as novel molecular target for stopping liver fibrosis from deterioration and may provide a new approach for the treatment of liver fibrosis.

\section{Declarations}

\section{Acknowledgments and Funding}

We would like to thank the anonymous reviewers for their helpful remarks. We wish to acknowledge Dr. XUE, Dr. Tan, Professor of Hai ZHENG and Hua Zhong University of Science and Technology for the help 
in interpreting the significance of the results of this study. This project was supported by the National Natural Science Foundation of China (NSFC) under grant No. 81071541.

\section{Competing Interests}

Not applicable, we declare that we have no financial and personal relationships with other individuals or organizations that can, in any way, inappropriately influence the results and interpretation of our work.

\section{Data availability statement}

The data that support the findings of this study are available from the corresponding author upon reasonable request.

\section{Authors' contributions}

YX conceived, designed, and supervised the study and revised the manuscript. JT performed the experiments and provided technical support. TY, QC, KJ and ZW analyzed the data and edited the manuscript. All authors have read, revised and approved the final version of the manuscript.

\section{Ethics approval and consent to participate}

The present study was approved by the Ethical Committee of Tongji Medical College, Huazhong University of Science and Technology, and the approval number was [2015] Ethical approval No. (273).

\section{References}

Anthony, B., Allen, J. T., Li, Y. S. S., \& McManus, D. P. (2010, Jul 21). Hepatic stellate cells and parasiteinduced liver fibrosis. Parasites \& Vectors, 3. <Go to ISI>://WOS:000282495100002

Blomhoff, R., \& Wake, K. (1991, 1991-Mar-01). Perisinusoidal stellate cells of the liver: important roles in retinol metabolism and fibrosis. FASEB journal : official publication of the Federation of American Societies for Experimental Biology, 5(3), 271-277. <Go to ISI>://MEDLINE:2001786

Domitrovic, R., Jakovac, H., Tomac, J., \& Sain, I. (2009, Dec 15). Liver fibrosis in mice induced by carbon tetrachloride and its reversion by luteolin. Toxicology and Applied Pharmacology, 241(3), 311-321. <Go to ISI>://WOS:000271841400008

Eng, F. J., \& Friedman, S. L. (2000, Jul). Fibrogenesis I. New insights into hepatic stellate cell activation: the simple becomes complex. American Journal of Physiology-Gastrointestinal and Liver Physiology, 279(1), G7-G11. <Go to ISI>://WOS:000088144700002

Forbes, S. J., \& Parola, M. (2011, Apr). Liver fibrogenic cells. Best Practice \& Research Clinical Gastroenterology, 25(2), 207-217. <Go to |S|>://WOS:000290926200003 
Forbes, S. J., Russo, F. P., Rey, V., Burra, P., Rugge, M., Wright, N. A., \& Alison, M. R. (2004, Apr). A significant proportion of myofibroblasts are of bone marrow origin in human liver fibrosis. Gastroenterology, 126(4), 955-963. <Go to |S|>://WOS:000220688100006

Friedman, S. L. (2000, Jan 28). Molecular regulation of hepatic fibrosis, an integrated cellular response to tissue injury. J Biol Chem, 275(4), 2247-2250. https://doi.org/10.1074/jbc.275.4.2247

Garrett-Sinha, L. A., Eberspaecher, H., Seldin, M. F., \& De Crombrugghe, B. $(1996,1996)$. A gene for a novel zinc-finger protein expressed in differentiated epithelial cells and transiently in certain mesenchymal cells. Journal of Biological Chemistry, 271(49), 31384-31390. https://doi.org/10.1074/jbc.271.49.31384

George, J., Roulot, D., Koteliansky, V. E., \& Bissell, D. M. (1999, Oct 26). In vivo inhibition of rat stellate cell activation by soluble transforming growth factor beta type II receptor: A potential new therapy for hepatic fibrosis. Proceedings of the National Academy of Sciences of the United States of America, 96(22), 12719-12724. <Go to ISI>://WOS:000083373000094

George, J., Wang, S. S., Sevcsik, A. M., Sanicola, M., Cate, R. L., Koteliansky, V. E., \& Bissell, D. M. (2000, Jan). Transforming growth factor-beta initiates wound repair in rat liver through induction of the EIIIAfibronectin splice isoform. American Journal of Pathology, 156(1), 115-124. <Go to ISI>://WOS:000084773300015

Ghiassi-Nejad, Z., \& Friedman, S. L. (2008, Dec). Advances in antifibrotic therapy. Expert Rev Gastroenterol Hepatol, 2(6), 803-816. https://doi.org/10.1586/17474124.2.6.803

Gonzalez, S. A., Fiel, M. I., Sauk, J., Canchis, P. W., Liu, R. C., Chiriboga, L., Yee, H. T., Jacobson, I. M., \& Talal, A. H. (2009, Feb). Inverse association between hepatic stellate cell apoptosis and fibrosis in chronic hepatitis C virus infection. Journal of Viral Hepatitis, 16(2), 141-148. <Go to ISI>://WOS:000262943400007

Gressner, A. M., Weiskirchen, R., Breitkopf, K., \& Dooley, S. (2002, Apr). Roles of TGF-beta in hepatic fibrosis. Frontiers in Bioscience-Landmark, 7, D793-D807. <Go to ISI>://WOS:000174600800009

Guyot, C., Lepreux, S., Combe, C., Doudnikoff, E., Bioulac-Sage, P., Balabaud, C., \& Desmouliere, A. (2006, Feb). Hepatic fibrosis and cirrhosis: The (myo)fibroblastic cell subpopulations involved. International Journal of Biochemistry \& Cell Biology, 38(2), 135-151. <Go to ISI>://WOS:000233864900001

Heymann, F., Trautwein, C., \& Tacke, F. (2009, Sep). Monocytes and Macrophages as Cellular Targets in Liver Fibrosis. Inflammation \& Allergy Drug Targets, 8(4), 307-318. <Go to ISI>://BCI:BCI201000003944

Jhandier, M. N., Kruglov, E. A., Lavoie, E. G., Sevigny, J., \& Dranoff, J. A. (2005, Jun 17). Portal fibroblasts regulate the proliferation of bile duct epithelia via expression of NTPDase2. Journal of Biological Chemistry, 280(24), 22986-22992. <Go to ISI>://WOS:000229741800053 
Kisseleva, T., Cong, M., Paik, Y., Scholten, D., Jiang, C. Y., Benner, C., Iwaisako, K., Moore-Morris, T., Scott, B., Tsukamoto, H., Evans, S. M., Dillmann, W., Glass, C. K., \& Brenner, D. A. (2012, Jun 12). Myofibroblasts revert to an inactive phenotype during regression of liver fibrosis. Proceedings of the National Academy of Sciences of the United States of America, 109(24), 9448-9453. <Go to ISI>://WOS:000305511300052

Kisseleva, T., Uchinami, H., Feirt, N., Quintana-Bustamante, O., Segovia, J. C., Schwabe, R. F., \& Brenner, D. A. (2006, Sep). Bone marrow-derived fibrocytes participate in pathogenesis of liver fibrosis. Journal of Hepatology, 45(3), 429-438. <Go to ISI>://WOS:000240531100014

Lee, U. E., \& Friedman, S. L. (2011, Apr). Mechanisms of hepatic fibrogenesis. Best Practice \& Research in Clinical Gastroenterology, 25(2), 195-206. <Go to ISI>://WOS:000290926200002

Maher, J. J., Zia, S., \& Tzagarakis, C. $(1994,1994)$. Acetaldehyde-induced stimulation of collagen synthesis and gene expression is dependent on conditions of cell culture: Studies with rat lipocytes and fibroblasts. Alcoholism Clinical and Experimental Research, 18(2), 403-409.

https://doi.org/10.1111/j.1530-0277.1994.tb00033.x

Minar, J. (1987, Jul). [Worldwide distribution of mosquitoes in the subfamily Anophelinae and its relation to the transmission of malaria]. Cesk Epidemiol Mikrobiol Imunol, 36(4), 244-251.

https://www.ncbi.nlm.nih.gov/pubmed/2888539 (Svetove rozsireni komaru podceledi Anophelinae a jeho vztah k prenosu malarie.)

Moreira, R. K. (2007, Nov). Hepatic stellate cells and liver fibrosis. Archives of Pathology \& Laboratory Medicine, 131(11), 1728-1734. <Go to ISI>://WOS:000250734600022

Novo, E., Cannito, S., Patemostro, C., Bocca, C., Miglietta, A., \& Parola, M. (2014, Apr 15). Cellular and molecular mechanisms in liver fibrogenesis. Archives of Biochemistry and Biophysics, 548, 20-37. <Go to ISI>://WOS:000334975300003

Ramachandran, P., \& Iredale, J. P. (2012, Jun). Macrophages: Central regulators of hepatic fibrogenesis and fibrosis resolution. Journal of Hepatology, 56(6), 1417-1419. <Go to ISI>://WOS:000304512300031

Rockey, D. C., Housset, C. N., \& Friedman, S. L. (1993, 1993-Oct). Activation-dependent contractility of rat hepatic lipocytes in culture and in vivo. The Journal of clinical investigation, 92(4), 1795-1804.

https://doi.org/10.1172/jci116769

Sako, T., Yuspa, S. H., Herald, C. L., Pettit, G. R., \& Blumberg, P. M. (1987, 1987-Oct-15). Partial parallelism and partial blockade by bryostatin 1 of effects of phorbol ester tumor promoters on primary mouse epidermal cells. Cancer research, 47(20), 5445-5450. <Go to ISI>://MEDLINE:2888531

Schnaper, H. W. (1995). Balance between matrix synthesis and degradation: A determinant of glomerulosclerosis [Article]. Pediatric Nephrology, 9(1), 104-111. https://doi.org/10.1007/BF00858986 
Senoo, H., Yoshikawa, K., Morii, M., Miura, M., Imai, K., \& Mezaki, Y. (2010, Dec). Hepatic stellate cell (vitamin A-storing cell) and its relative - past, present and future. Cell Biology International, 34(12), 12471272. https://doi.org/10.1042/Cbi20100321

Shields, J. M., Christy, R. J., \& Yang, V. W. (1996, 1996). Identification and characterization of a gene encoding a gut-enriched Krueppel-like factor expressed during growth arrest. Journal of Biological Chemistry, 271(33), 20009-20017. https://doi.org/10.1074/jbc.271.33.20009

Suarez-Cuenca, J. A., de Sanchez, V. C., Aranda-Fraustro, A., Sanchez-Sevilla, L., Martinez-Perez, L., \& Hernandez-Munoz, R. (2008, Jul). Partial hepatectomy-induced regeneration accelerates reversion of liver fibrosis involving participation of hepatic stellate cells. Experimental Biology and Medicine, 233(7), 827839. <Go to ISI>://WOS:000257154500006

Tsukamoto, H. (2005, Nov). Adipogenic phenotype of hepatic stellate cells. Alcoholism-Clinical and Experimental Research, 29(11), 132s-133s. https://doi.org/10.1097/01.alc.0000189279.92602.f0

Wang, Y. Z., Li, J. L., Wang, X., Sang, M., \& Ho, W. Z. (2013, Aug). Hepatic stellate cells, liver innate immunity, and hepatitis C virus. Journal of Gastroenterology and Hepatology, 28, 112-115. <Go to ISI>://WOS:000329463400019

Wells, R. G., Kruglov, E., \& Dranoff, J. A. (2004, Feb 13). Autocrine release of TGF-beta by portal fibroblasts regulates cell growth. Febs Letters, 559(1-3), 107-110. <Go to ISI>://WOS:000188970100019

Zeisberg, M., Yang, C. Q., Martino, M., Duncan, M. B., Rieder, F., Tanjore, H., \& Kalluri, R. (2007, Aug 10). Fibroblasts derive from hepatocytes in liver fibrosis via epithelial to mesenchymal transition. Journal of Biological Chemistry, 282(32), 23337-23347. <Go to ISI>://WOS:000248577500034

Zhao, Q., Qin, C. Y., Zhao, Z. H., Fan, Y. C., \& Wang, K. (2013, Jan). Epigenetic Modifications in Hepatic Stellate Cells Contribute to Liver Fibrosis. Tohoku Journal of Experimental Medicine, 229(1), 35-43. <Go to ISI>://WOS:000313932400006

\section{Figures}




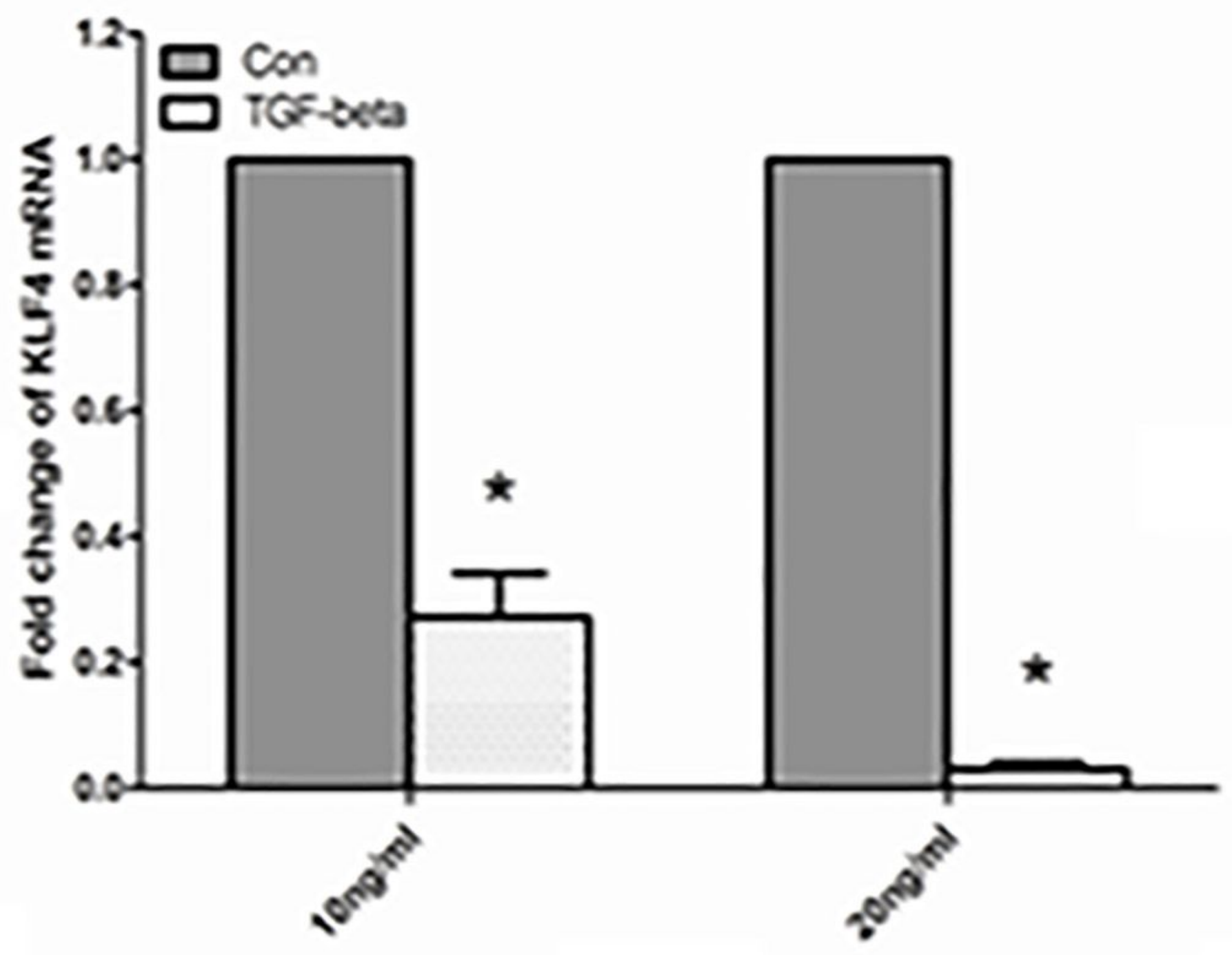

Figure 1

KLF4 mRNA expression in LX-2 cells cultured with different concentrations of TGF- $\beta$ was decreased after 24 hours. 

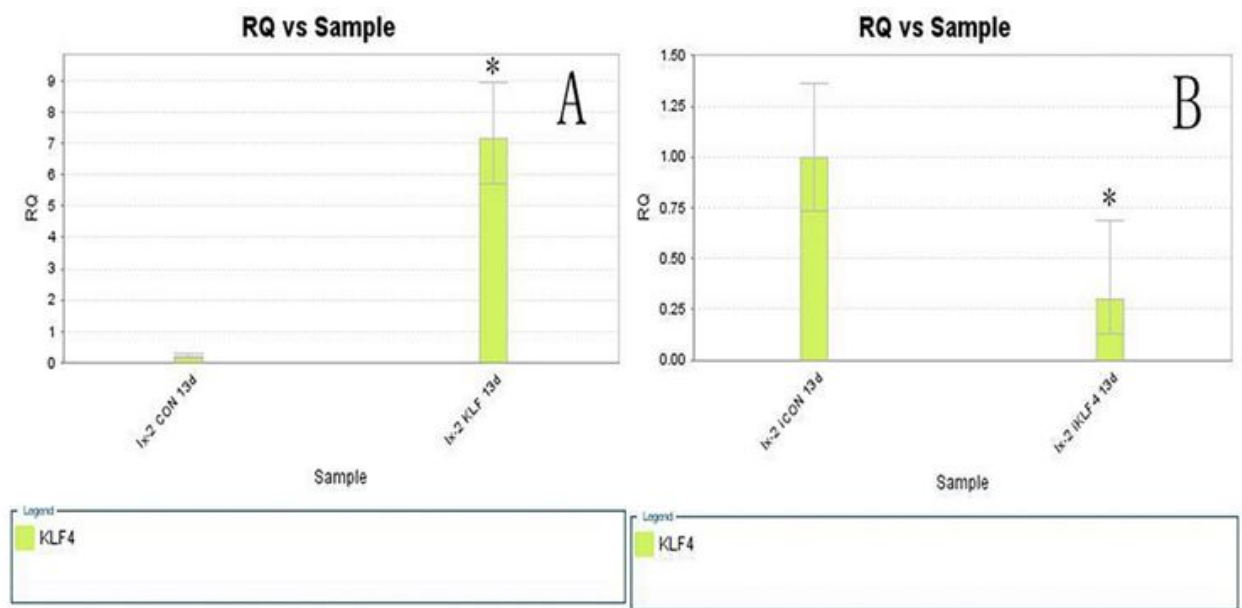

KLF4

C Con+ LX-2

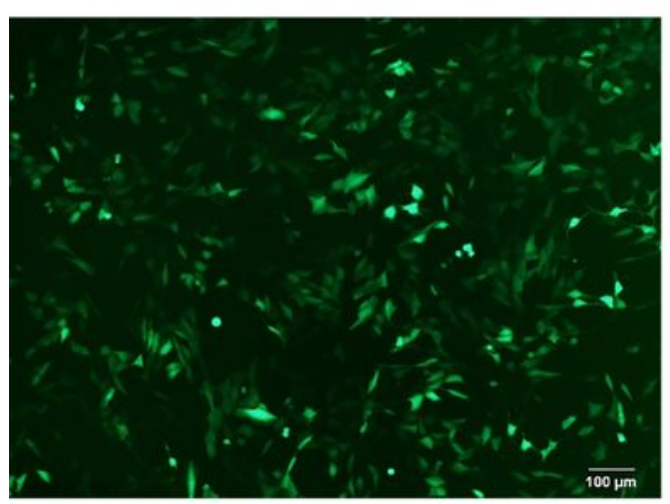

E iCon LX-2
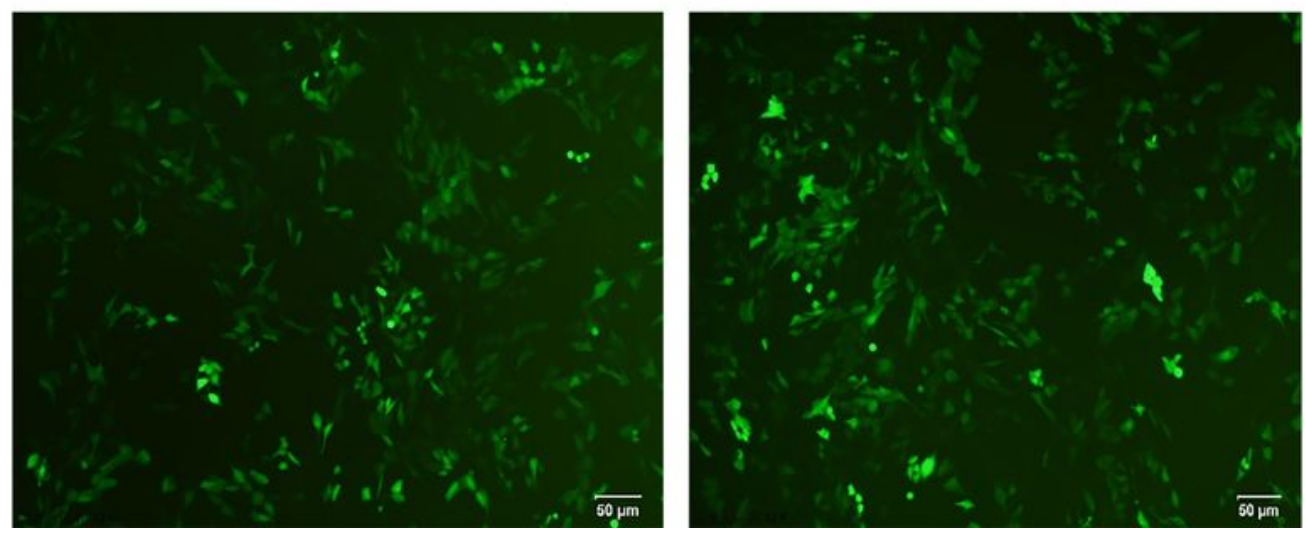

\section{Figure 2}

Transfection of LX-2 cells with lentiviral vector. A: LX-2 cells overexpressed KLF4. B囚Silencing of KLF4 expression in LX-2 cells. C: KLF4 overexpression group. 
A
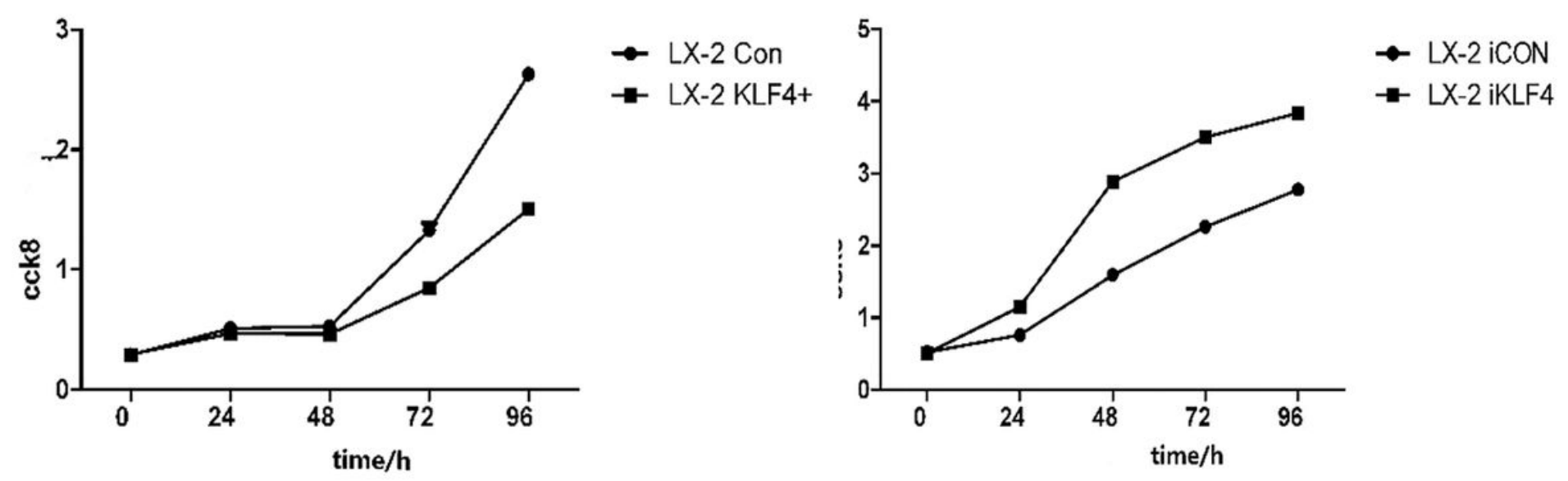

Figure 3

CCK8 detection of LX-2 cell proliferation 

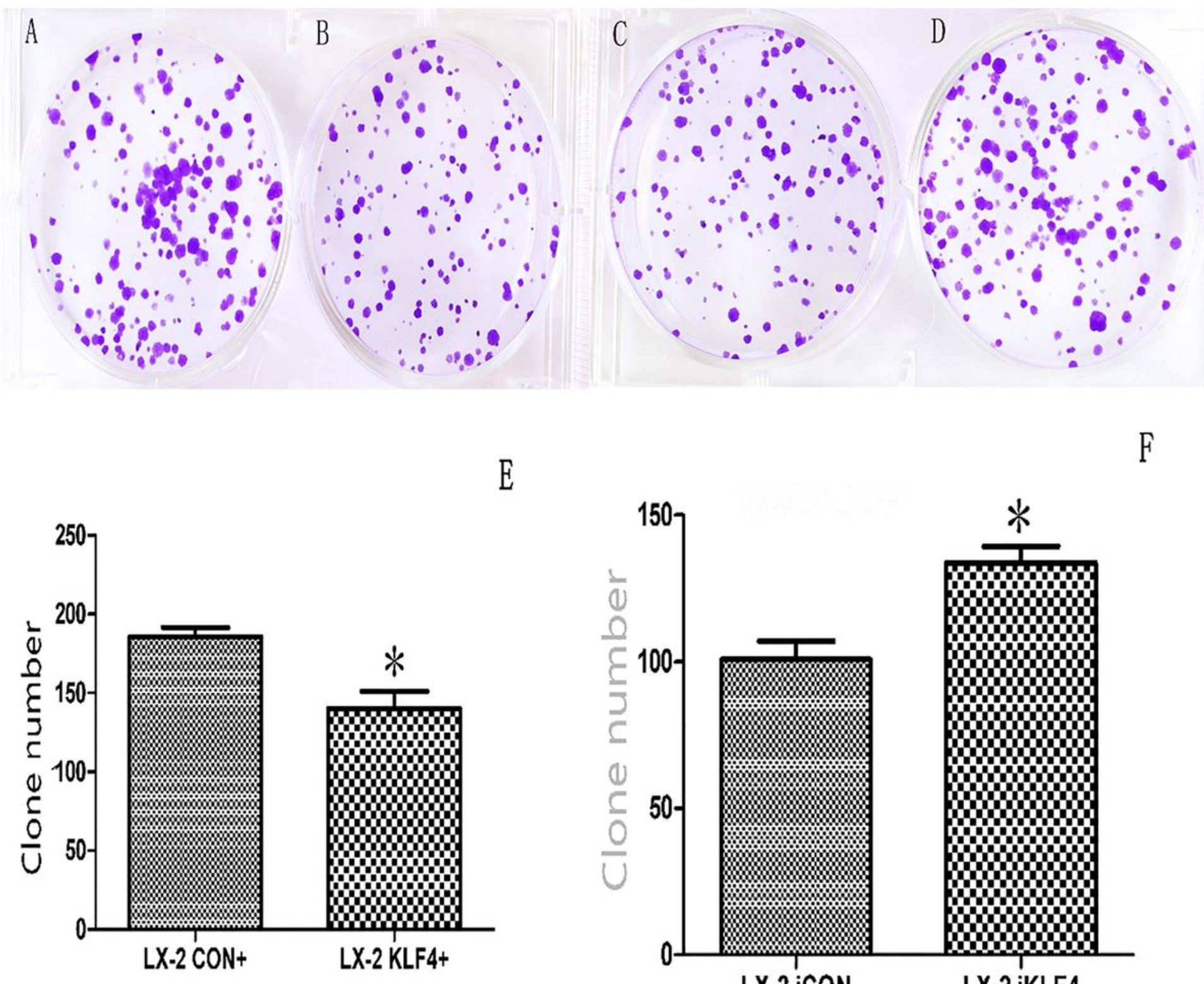

E

F

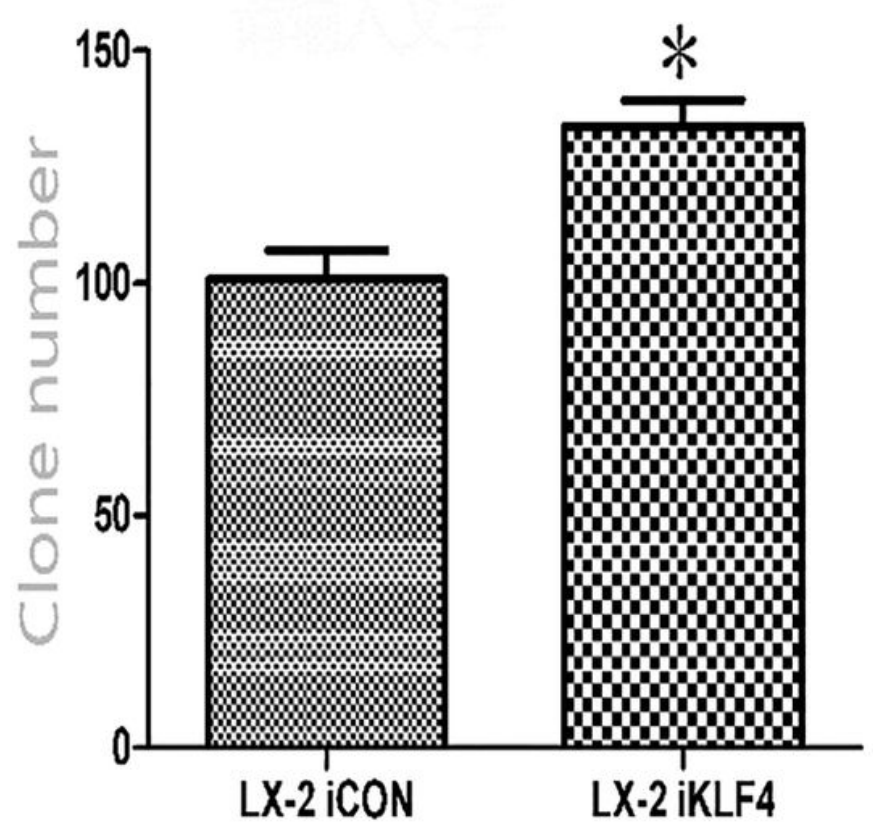

Figure 4

Plate clone map and chart. 


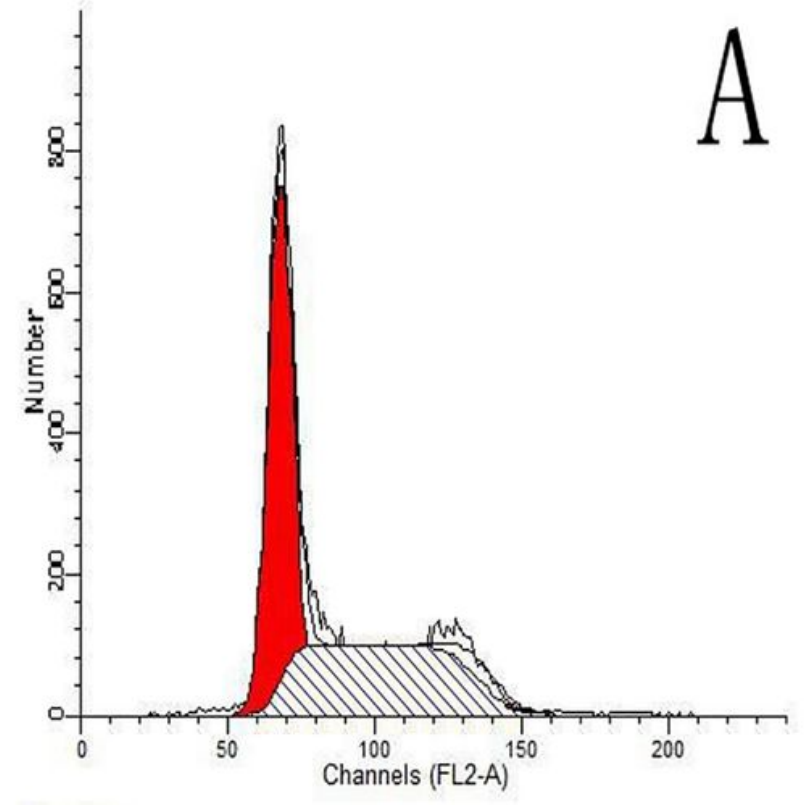

Data.001

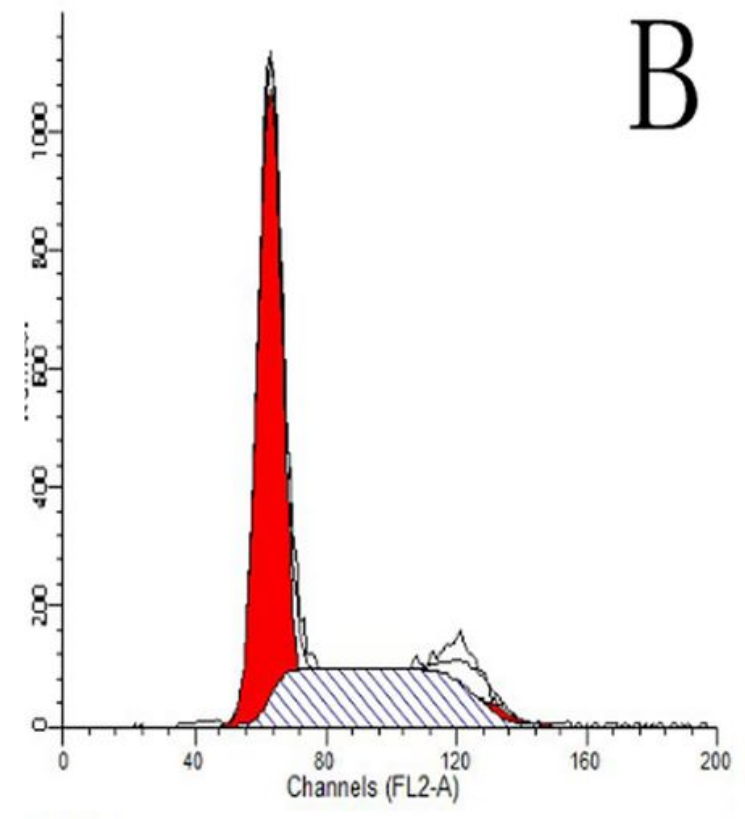

ata. 002

\section{Figure 5}

Flow cytometrical detection of cell cycle. A. The control group transfect by empty virus. B. The LX-2 group up-regulated KLF4 expression.

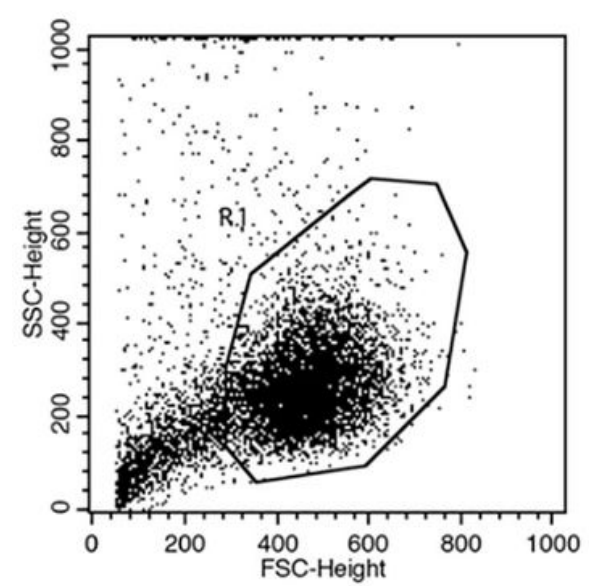

File: A

Gated Events: 8525

$X$ Parameter: 7AAD (Log)

\begin{tabular}{crrrrr} 
Quad & Events & $\%$ Gated & $\%$ Total & X Mean & Y Mean \\
\hline UL & 37 & 0.43 & 0.36 & 9.35 & 93.93 \\
UR & 101 & 1.18 & 0.98 & 90.01 & 346.89 \\
LL & 7867 & 92.28 & 76.68 & 6.15 & 4.71 \\
LR & 520 & 6.10 & 5.07 & 65.69 & 12.68
\end{tabular}

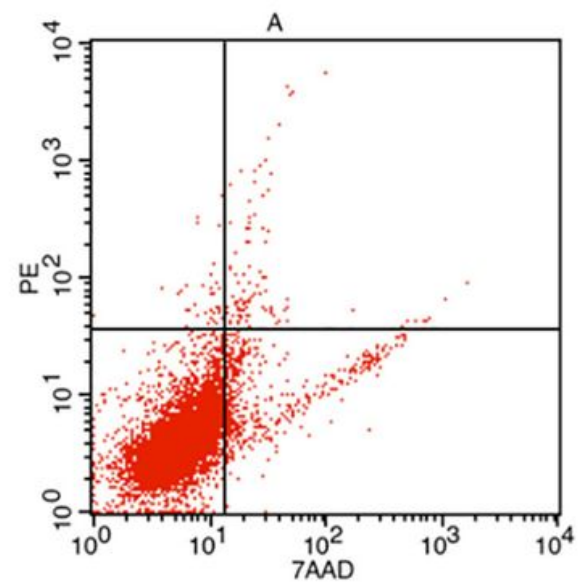

Gate: G1

Total Events: 10260

Y Parameter: PE (Log)
File: B

Gated Events: 16901

X Parameter: 7AAD (Log)

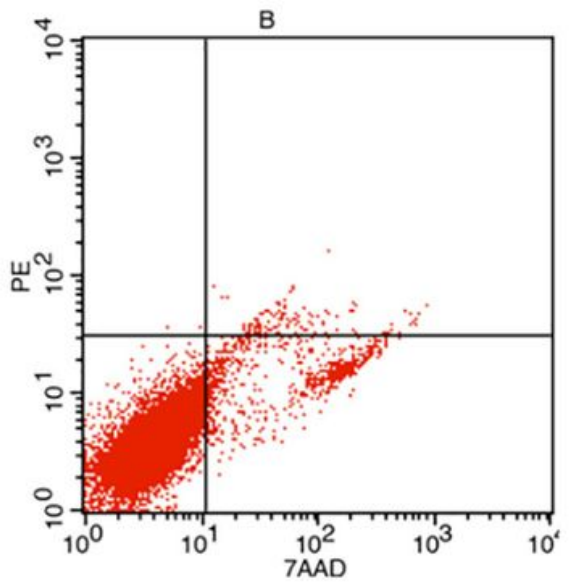

Gate: G1

Total Events: 20000

Y Parameter: PE (Log)

\begin{tabular}{crrrrrr} 
Quad & Events & $\%$ Gated & $\%$ Total & X Mean & Y Mean \\
\cline { 1 - 4 } UL & 2 & 0.01 & 0.01 & 7.35 & 35.71 \\
UR & 120 & 0.71 & 0.60 & 119.07 & 42.99 \\
LL & 15969 & 94.49 & 79.84 & 4.58 & 4.27 \\
LR & 810 & 4.79 & 4.05 & 95.80 & 15.26
\end{tabular}

Figure 6 
Flow cytometrical detection of apoptosis of cells with KLF4 overexpression

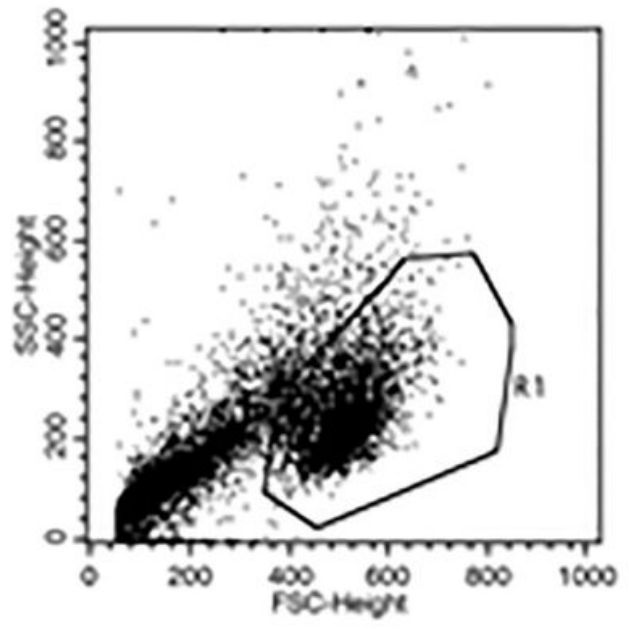

File: $A$

Gated Events: 10517

X Paramoter: 7AAD (Log)

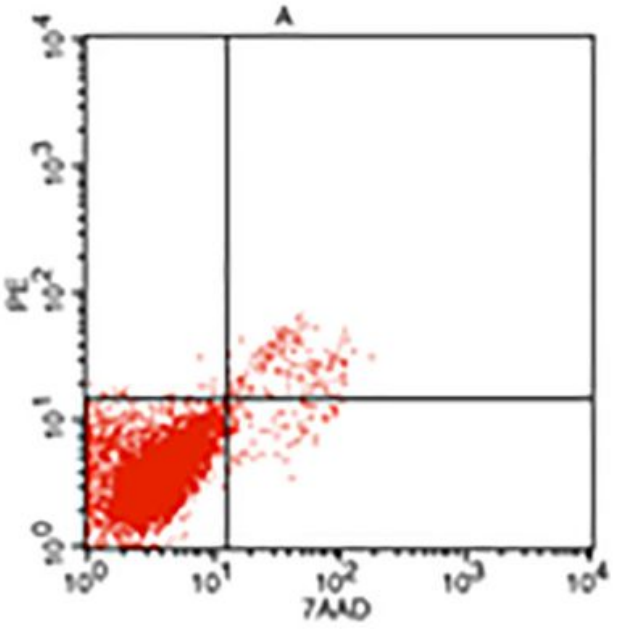

Gato: G1

Total Events: 20000

Y Paramoter: PE (Log)
File: 8

Gated Events: 12571

X Parameser: $7 A A D(L O g)$

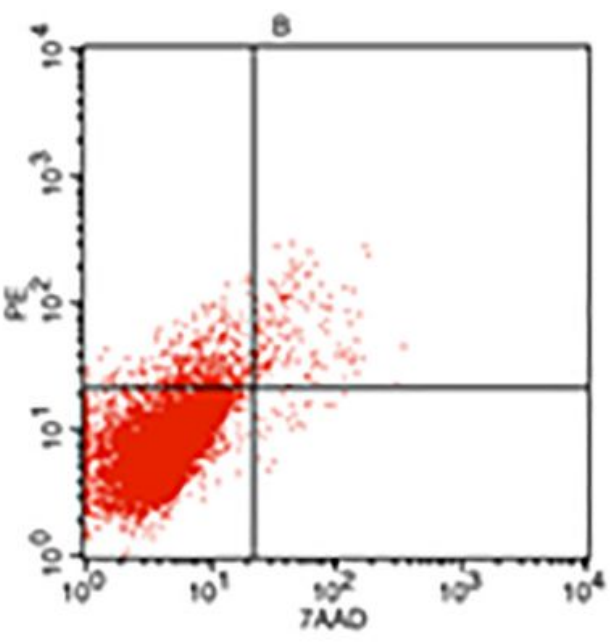

Ga:e: G1

Toes Events: 20000

Y Parameter: PE (Log)

\begin{tabular}{|c|c|c|c|c|c|c|c|c|c|c|c|}
\hline Juad & Events & $\%$ Ga:ed & $\%$ Total & $x$ Mean & Y Mean & Cuasd & Events & $\%$ Gatod & $\%$ Total & $x$ Mosn & Y Moan \\
\hline UL & 25 & 0.24 & 0.12 & 5.11 & 16.89 & UL & 553 & 4.40 & 2.77 & 9.39 & 3328 \\
\hline UR & 193 & 84 & 0.97 & 46.4 & 28.93 & UR & 160 & 1.27 & 0.80 & 55.28 & 7243 \\
\hline $\mathrm{u}$ & 10193 & 96.92 & 50.97 & 4.37 & 4.41 & u & 11835 & 94.15 & 59.17 & 4.97 & 8.28 \\
\hline LR & 106 & 1.01 & 0.53 & 32.61 & 9.55 & LR & 23 & 0.18 & 0.11 & 45.77 & 1255 \\
\hline
\end{tabular}

Figure 7

Flow cytometrical detection of apoptosis in KLF4 inhibition group. 

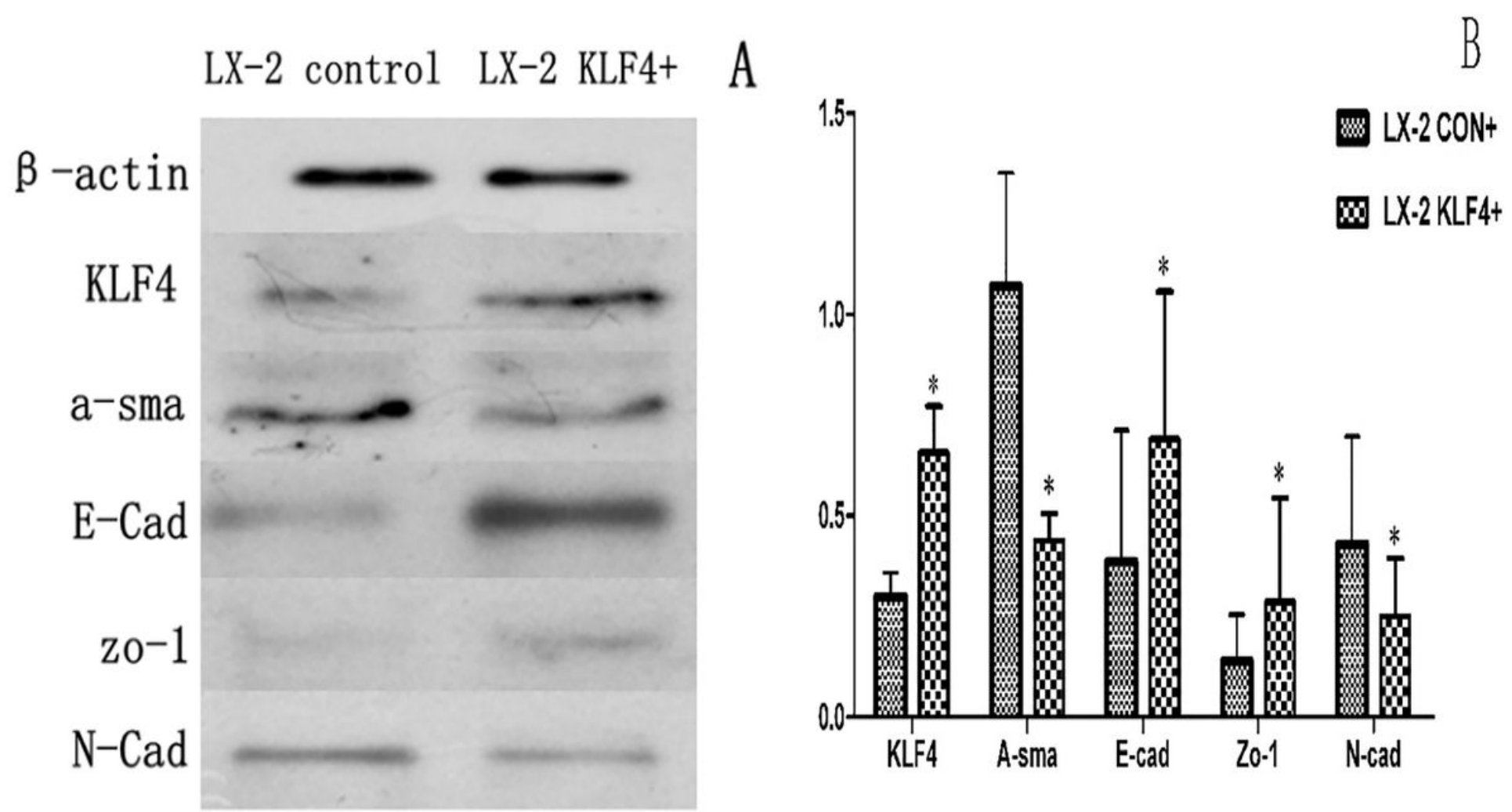

Figure 8

Western blotting results

\section{Supplementary Files}

This is a list of supplementary files associated with this preprint. Click to download.

- renamed622e8.pdf 